\title{
Nanoscale
}

PAPER
View Article Online

View Journal | View Issue
Check for updates

Cite this: Nanoscale, 2018, 10, 5965

\section{Graphene oxide size and oxidation degree govern its supramolecular interactions with siRNA $\uparrow$}

\author{
Giacomo Reina, (D)*a Ngoc Do Quyen Chau, ${ }^{a}$ Yuta Nishina (D) ${ }^{\text {b,c }}$ and \\ Alberto Bianco (iD *a
}

Several studies have demonstrated the ability of graphene oxide (GO) to efficiently adsorb small-interfering RNA (siRNA) on its surface and to transport it into cells. However, studies on whether and how siRNA interacts with GO are still inconclusive. In this context, understanding the interaction between GO and siRNA is fundamental to design new efficient gene silencing tools. In this work, the interactions between GO and siRNA molecules were systematically investigated. We focused on how the GO size, oxygenated groups present on the surface and chemical functionalization affect the double helix siRNA structure, using gel electrophoresis, UV-Vis spectroscopy, fluorescence resonance energy transfer (FRET) and circular dichroism (CD). We found that the siRNA secondary structure was clearly altered by the interaction with GO flakes. In addition, we were able to correlate the double strand damage with the size and the oxygenated groups present on the GO sheets. Finally, we demonstrated that GO functionalized with low molecular weight polyethyleneimine (PEI, $800 \mathrm{Da}$ ) is able to protect siRNA from structural modifications. We believed that this research effort will improve our understanding of the behavior of GO/siRNA complexes, and thus facilitate the design of appropriate bio/nanointerfaces and new efficient gene silencing systems.

Received 12th January 2018

Accepted 11th February 2018

DOI: $10.1039 /$ c8nr00333e

rsc.li/nanoscale specific protein expression. ${ }^{5,6}$ However, the delivery of siRNA to target cells has inherent drawbacks mainly due to the siRNA instability, and low transfection efficiency, biocompatibility and biodistribution. ${ }^{7}$ Therefore, the development of effective and nontoxic delivery systems is crucial to overcome the barrier between the potential of gene silencing and a real therapeutic application.

Due to its unique physicochemical and structural properties, its electrical and optical characteristics and biodegradability, graphene and its derivatives have attracted increasing interest as a prominent potential vector in gene delivery. ${ }^{8-11}$ In particular, graphene oxide, the oxidized and water soluble form of graphene, offers a wide opportunity to load both hydrophilic and hydrophobic molecules and different target species on its large surface area. ${ }^{12}$ Studies of the interactions of GO with nucleic acids have been substantially enhanced over the past few years for the application in biosensing, diagnostics and biomedicine. ${ }^{13-15}$ Generally, the binding between GO and single strand DNA (ssDNA) or single strand RNA (ssRNA) is ascribed either to the $\pi-\pi$ stacking or the hydrogen bonding and van der Waals forces. ${ }^{16}$ In the case of double strand DNA (dsDNA), Lei et al. ${ }^{17}$ have figured out that DNA can bind GO to form a dsDNA/GO complex in the presence of a high concentration of salts via electrostatic interactions. Liu et $a l .{ }^{18}$ also reported the salt-controlled assembly of graphene sheets to capture dsDNA in high salt concentration. In contrast, Tang et al., ${ }^{19}$ by measuring the quenching effect between 
a fluorescently-labeled dsDNA and GO, proved that there is no effect of salt on fluorescence change, thus excluding the electrostatic interactions as the major driving force for dsDNA on the surface of GO. Moreover, this study explored for the first time the partial deformation of dsDNA incubated with GO. The GO induced deformation of dsDNA had been previously predicted by molecular dynamics simulation studies, showing that the hydrophobic $\pi-\pi$ stacking between GO and dsDNA had a dominant role in the DNA structure alteration. ${ }^{20}$ On the other hand, Ren et $a .^{21}$ suggested that GO can intercalate efficiently into DNA molecules and enhance the double strand cleavage activity of copper ions via a mechanism based on an oxidative and hydrolytic effect. Besides, another study proved that the DNA cleavage induced by GO and reduced GO with a different $\mathrm{Cu}^{2+}$ complex was trigged by interaction of GO with DNA. ${ }^{22}$ Despite these studies, understanding deeply how the conformation of double strand siRNA is affected by GO remains ambiguous. A thorough comprehension of the mechanism of interaction between GO and siRNA is crucial for application of GO in future clinical settings. For these reasons, we performed various spectroscopic investigations demonstrating that non-functionalized GO significantly alters the double strand native structure. In particular, small GO flakes seem to intercalate inside the double strand, breaking some of the base to base hydrogen bonds (H-bonds). On the other hand, large GO flakes alter mainly the secondary structure geometry interacting mostly with the helix groove and causing the winding angle relaxation. Interestingly, PEI functionalized GO is able to avoid siRNA double strand structural modifications and to sensibly enhance siRNA adsorption on GO sheets.

\section{Materials and methods}

\subsection{Materials}

Polyethyleneimine (PEI) (MW $800 \mathrm{Da}$ ), was purchased from Sigma Aldrich. The solvents were obtained from commercial suppliers and used without purification. Water was purified using a Millipore filter system MilliQ ${ }^{\circledR}$ and free endotoxin Polisseur Biopak®. When stated, suspensions were sonicated in a water bath $(20 \mathrm{~W}, 40 \mathrm{kHz})$. The filtration and dialysis membranes were purchased from Millipore and Spectrum Laboratories, Inc., respectively.

In this experiment, Yakima labeled siRNA (sUGC-GCU-ACG-AUC-GACGAU-G55), siRNA labeled at the $5^{\prime}$ position with Alexa Fluor ${ }^{\circledR} 546$, siRNA with one strand labeled at the $5^{\prime}$ position with Alexa Fluor ${ }^{\circledR} 546$ (donor) and the other strand labeled at the $5^{\prime}$ position with Alexa Fluor ${ }^{\circledR 6} 67$ (acceptor), siRNA labeled at the $5^{\prime}$ position with $\mathrm{Cy}{ }^{\circledR} 3$, and siRNA with one strand labeled at the $5^{\prime}$ position with $\mathrm{Cy} 囚 3$ (donor) and the other strand marked at the $3^{\prime}$ position with Cy®5 (acceptor) were purchased from Eurogentec.

\subsection{Instruments}

Thermogravimetric analysis (TGA) was performed on a TGA1 (Mettler Toledo) apparatus from $30{ }^{\circ} \mathrm{C}$ to $900{ }^{\circ} \mathrm{C}$ with a ramp of $10{ }^{\circ} \mathrm{C} \mathrm{min}^{-1}$ under $\mathrm{N}_{2}$ using a flow rate of $50 \mathrm{~mL} \mathrm{~min}^{-1}$ and platinum pans. X-ray Photoelectron Spectroscopy (XPS) analyses were performed on a Thermo Scientific K-Alpha X-ray photoelectron spectrometer with a basic chamber pressure of $10^{-8}-10^{-9}$ bar with an anode using $\mathrm{Al} \mathrm{K} \alpha$ radiation $(h \nu=$ $1486.6 \mathrm{eV})$. The C (1s) photoelectron binding energy was set at $284.5 \pm 0.2 \mathrm{eV}$ and used as a reference for calibrating the other peak positions. The samples were analyzed as powder. A spot size of $400 \mu \mathrm{m}$ was used. The survey spectra are the average of 10 scans with a pass energy of $200.00 \mathrm{eV}$ and a step size of $1 \mathrm{eV}$. The high-resolution spectra are an average of 10 scans with a pass energy of $50 \mathrm{eV}$ and a step size of $0.1 \mathrm{eV}$. An ion gun was turned on during analysis. For each sample, the analysis was repeated three times. X-ray diffraction was carried out by using a PANalytical Co. X'Pert PRO using $\mathrm{Cu}$ K $\alpha$ radiation $(\lambda=1.541 \AA)$ in the $2 \theta$ range of $5-20^{\circ}$. The operating tube current and voltage were $40 \mathrm{~mA}$ and $40 \mathrm{kV}$, respectively. Ozone treatment was performed using an ozone generator (Ozone Wave Pro, Syoken Co. Ltd). SEM measurement was performed on a SEM S-5200 (Hitachi High Technologies Corporation, Tokyo, Japan). UV-Vis absorption spectra were recorded on a Cary 5000 UV-Vis-NIR spectrophotometer and were corrected for the baseline and the solvent. Steady-state luminescence experiments were performed with a Fluoromax-4 spectrofluorometer (Horiba-Jobin-Yvon Inc.). Emission spectra were corrected for the monochromator and detector efficiency and for the light source intensity (450 W xenon arc lamp), employing standard correction files and recording the reference signal. Circular dichroism (CD) spectra have been recorded with a J-810 Jasco spectropolarimeter. Each spectrum was recorded at $1 \mathrm{~nm}$ resolution after 16 accumulations from 190 to $350 \mathrm{~nm}$. Filtration was performed using a Sterile Millex® $(13 \mathrm{~mm})$ filter unit with a Durapore ${ }^{\circledR}$ membrane and a pore size of $0.22 \mu \mathrm{m}$. HPLC analyses were performed on a Waters HPLC system (Waters Co., Milford, MA, USA) equipped with a reversedphase C-18 column system with an analytical C18 column (EC 150/4.6 NUCLEOSIL 100-5 C18 length: $150 \mathrm{~mm}$, ID: $4.6 \mathrm{~mm}$, $5 \mu \mathrm{m})$. The following solvent system was used: solvent A, $0.1 \mathrm{M}$ trimethylammonium acetate at $\mathrm{pH} 7.5$ and solvent $\mathrm{B}$, acetonitrile. Gradient elution was applied at a flow rate of 2 $\mathrm{mL} \min ^{-1}$ for $60 \mathrm{~min}$. The chromatograms were recorded following the absorption maximum of Alexa Fluor®647-labeled siRNA at $650 \mathrm{~nm}$.

\subsection{Preparation of graphene oxide with different oxygen percentages and their PEI functionalization}

Large graphene oxide $\left(\mathrm{GO}_{\mathrm{L}}\right)$ was synthesized following the modified Hummers method by the group of Nishina ${ }^{23}$ and obtained as an aqueous dispersion of GO. Small graphene oxide $\left(\mathrm{GO}_{\mathrm{S}}\right)$ was obtained from the company Nanoinnova as a powder. Reduced GO with different oxygen percentages was synthesized as mentioned in our previous report. ${ }^{24}$ Briefly, $\mathrm{rGO}_{\mathrm{L}}$ and $\mathrm{rGO}_{\mathrm{S}}$ were obtained from $\mathrm{GO}_{\mathrm{L}}$ and $\mathrm{GO}_{\mathrm{S}}$, respectively, heated and refluxed at $100{ }^{\circ} \mathrm{C}$ for 5 days with continuous stirring, washed with dimethylformamide (DMF), methanol $(\mathrm{MeOH})$, and dichloromethane (DCM), dialyzed 
against Milli-Q water and lyophilized. $\mathrm{rGO}_{\mathrm{L}}-\mathrm{O}_{3}$ and $\mathrm{rGO}_{\mathrm{S}}-\mathrm{O}_{3}$ were obtained by fluxing $\mathrm{O}_{3}$, which was generated from an air source, to a suspension of $\mathrm{rGO}_{\mathrm{L}}$ and $\mathrm{rGO}_{\mathrm{S}}$, respectively, in

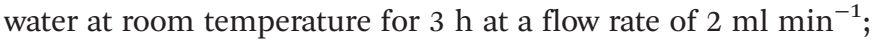
then the products were washed with $\mathrm{DMF}, \mathrm{MeOH}$, and DCM, dialyzed against Milli-Q water and lyophilized. PEI functionalized GO/rGO was obtained after an addition of PEI to a suspension of the corresponding $\mathrm{GO} / \mathrm{rGO}$ with a mass ratio of $1: 1$. The reaction mixture was stirred for 3 days, then centrifuged until the $\mathrm{pH}$ of the supernatant was neutral. Finally PEI functionalized GO/rGO was dialyzed against water (Spectra/ Por® dialysis membrane MWCO 12 000-14 $000 \mathrm{Da}$ ) for 3 days and lyophilized.

\subsection{Evaluation of siRNA/GO complexation by agarose gel electrophoresis mobility assay}

A solution of double-stranded Yakima-labeled siRNA $(C=$ $0.1 \mu \mathrm{g} \mu \mathrm{l}^{-1}$ ) was kept constant at $2 \mu \mathrm{l}$ in every mass ratio. The suspensions of different GO and PEI-GO in Milli-Q ${ }^{\circledR}$ RNAsefree water were prepared corresponding to each mass ratio $\mathrm{GO} /$ siRNA required for the complexation with siRNA. The suspensions were kept at room temperature for $30 \mathrm{~min}$ to allow the formation of the complexes. After staining with Orange DNA loading dye $(6 \times$, Thermo Fisher Scientific) (total loading sample volume $=30 \mu \mathrm{L}$ ), the suspensions were added to $2 \%$ agarose containing GelRed (Biotium, USA) followed by electrophoresis in Tris-Acetate-EDTA (TAE) buffer at $100 \mathrm{mV}$ for $30 \mathrm{~min}$. The gels were then visualized under UV light using the Gel Doc ${ }^{\mathrm{TM}}$ EZ Imager - Bio-Rad and Image lab software. The signal of each experiment was normalized to the signal of the free siRNA as a control and subtracted by the background signal by using the ImageJ program. Each experiment was repeated at least three times.

\subsection{Preparation of the solutions for spectroscopic analyses}

For dynamic equilibrium experiments using CD, $180 \mu \mathrm{l}$ of siRNA labeled Yakima $\left(C=0.1 \mu \mathrm{g} \mu \mathrm{l}^{-1}, m=18 \mu \mathrm{g}\right)$ and a suspension of $12 \mu \mathrm{l}$ of GO ( $\left.C=3 \mu \mathrm{g} \mu \mathrm{l}^{-1}, m=36 \mu \mathrm{g}\right)$ (GO/siRNA ratio: 2) were added to $258 \mu \mathrm{l}$ of Milli-Q ${ }^{\circledR}$ RNAse-free water. The helicity was calculated by normalizing the signal to the cuvette length $(0.2 \mathrm{~cm})$ and the molar concentration.

For CD and UV, to $180 \mu \mathrm{l}$ of siRNA labeled Yakima $(C=$ $\left.0.1 \mu \mathrm{g} \mu \mathrm{l}^{-1}, m=18 \mu \mathrm{g}\right), 270 \mu \mathrm{l}$ of Milli-Q ${ }^{\circledR}$ RNAse-free water was added to obtain $450 \mu \mathrm{l}$ of siRNA solution $\left(C^{\prime}=40 \mu \mathrm{g} \mathrm{ml}^{-1}\right)$ as a control. A calibration of siRNA signal in UV with concentrations from $10 \mu \mathrm{g} \mathrm{ml} \mathrm{m}^{-1}$ to $50 \mu \mathrm{g} \mathrm{ml} \mathrm{m}^{-1}$ was also performed. At a mass ratio of GO/siRNA equal to 20 , to $180 \mu \mathrm{l}$ of $\operatorname{siRNA}(C=$ $\left.0.1 \mu \mathrm{g} \mu \mathrm{l}^{-1}, m=18 \mu \mathrm{g}\right)$, a suspension of $72 \mu \mathrm{l}$ of $\mathrm{GO}(C=5$ $\left.\mu \mathrm{g} \mu \mathrm{l}^{-1}, m=360 \mu \mathrm{g}\right)$ and $198 \mu \mathrm{l}$ of Milli-Q ${ }^{\circledR}$ RNAse-free water were added to obtain a solution of siRNA at the same concentration as the control. After the interaction of GO with siRNA, the helicity was calculated by normalizing the signal to the cuvette length $(0.2 \mathrm{~cm})$ and the molar concentration obtained from the Yakima signal at $523 \mathrm{~nm}$ in UV.

For the FRET analysis, to $60 \mu \mathrm{l}$ of each siRNA (siRNA labeled Cy3, siRNA labeled Cy3/Cy5, siRNA labeled Alexa
Fluor®546, siRNA labeled Alexa Fluor®546/Alexa Fluor®647, $\left.C=0.1 \mu \mathrm{g} \mu^{-1}, m=6 \mu \mathrm{g}\right), 90 \mu \mathrm{l}$ of Milli-Q ${ }^{\circledR}$ RNAse-free water was added to obtain separately $150 \mu \mathrm{l}$ of siRNA solution $\left(C^{\prime}=\right.$ $40 \mu \mathrm{g} \mathrm{ml}^{-1}$ ) as a control. At a mass ratio of GO/siRNA equal 20, to $60 \mu \mathrm{l}$ of siRNA $\left(C=0.1 \mu \mathrm{g} \mu \mathrm{l}^{-1}, m=6 \mu \mathrm{g}\right)$, a suspension of $24 \mu \mathrm{l}$ of GO $\left(C=5 \mu \mathrm{g} \mu \mathrm{l}^{-1}, m=120 \mu \mathrm{g}\right)$ and $66 \mu \mathrm{l}$ of Milli-Q ${ }^{\circledR}$ RNAse-free water were added to obtain a solution of siRNA at the same concentration as the control. After the interaction of siRNA with GO, the filtered siRNA solutions were excited at $535 \mathrm{~nm}$ and the maximum was recorded at 570 and $575 \mathrm{~nm}$ for Alexa 546 and Cy3, respectively. The FRET efficiency was calculated by normalizing for the absorbance of each solution at $535 \mathrm{~nm}$ (measured by UV-Vis). Statistical analyses were performed using an unpaired $t$-test.

\subsection{Preparation of the solutions for HPLC analyses}

For HPLC analysis, $100 \mu \mathrm{l}$ of Milli-Q ${ }^{\circledR}$ RNAse-free water was added to $100 \mu \mathrm{l}$ of siRNA (siRNA labeled Alexa Fluor®647, $C=$ $0.1 \mu \mathrm{g} \mu \mathrm{l}^{-1}, m=10 \mu \mathrm{g}$ ) to obtain $200 \mu \mathrm{l}$ of siRNA solution as a control. For the denaturation of siRNA, $100 \mu \mathrm{l}$ of a solution of $2 \%$ of formaldehyde in Milli-Q ${ }^{\circledR}$ RNAse-free water was added to $100 \mu \mathrm{l}$ of siRNA (siRNA labeled Alexa Fluor ${ }^{\circ} 647, C=0.1$ $\left.\mu \mathrm{g} \mu \mathrm{l}^{-1}, m=10 \mu \mathrm{g}\right)$ to obtain $200 \mu \mathrm{l}$ of final solution. Subsequently, the solution was heated at $95^{\circ} \mathrm{C}$ for $1 \mathrm{~min}$ and immediately cooled down using an ice bath. In the case of the analysis of the sample prepared at the mass ratio of GO/siRNA equal to 20 , a suspension of $66.7 \mu \mathrm{l}$ of GO $\left(C=3 \mu \mathrm{g} \mu \mathrm{l}^{-1}, m=\right.$ $200 \mu \mathrm{g})$ and $33.3 \mu \mathrm{l}$ of Milli-Q ${ }^{\circledR}$ RNAse-free water were added to $100 \mu \mathrm{l}$ of siRNA $\left(C=0.1 \mu \mathrm{g} \mu \mathrm{l}^{-1}, m=10 \mu \mathrm{g}\right)$ to obtain a solution of siRNA at the same concentration as the control. After the interaction of siRNA with GO, the filtered siRNA solutions were injected in HPLC.

\section{Results and discussion}

\subsection{Preparation and characterization of non-functionalized and PEI-functionalized GO materials}

The aim of this work was to elucidate the mechanism of the interaction between siRNA molecules and GO flakes. For this purpose the lateral size, oxygenated groups and functionalization effects were taken into consideration. We decided to use two different samples of academic (named $\mathrm{GO}_{\mathrm{L}}$ ) and commercial (named $\mathrm{GO}_{\mathrm{S}}$ ) sources, both prepared by the Hummers method. ${ }^{23}$ The two GO materials are prepared by a slightly different process, as they differ in the percentage of oxygenated groups and lateral sizes. The lateral sizes of two GO samples correspond to $300 \pm 200 \mathrm{~nm}$ in the case of $\mathrm{GO}_{\mathrm{S}}$ (Fig. S1 in the $\mathrm{ESI} \dagger$ ) and $3 \pm 1 \mu \mathrm{m}$ for $\mathrm{GO}_{\mathrm{L}}$. To understand how the oxygenated groups affect the siRNA structure, we decided to perform a reduction and a subsequent epoxidation of the graphene oxide materials. Reduced $\mathrm{GO}_{\mathrm{S}}\left(\mathrm{rGO}_{\mathrm{S}}\right)$ and $\mathrm{GO}_{\mathrm{L}}\left(\mathrm{rGO}_{\mathrm{L}}\right)$ were prepared under green conditions without using any toxic reagents. Epoxidation reaction was performed on $\mathrm{rGO}_{\mathrm{S}}$ and $\mathrm{rGO}_{\mathrm{L}}$ using $\mathrm{O}_{3}$ under the same conditions reported before. ${ }^{24}$ In addition, aiming to study the effect of amino functionali- 
Table 1 Different GO materials used in this study and C/O ratios of non-functionalized GO obtained by XPS analysis

\begin{tabular}{|c|c|c|c|c|c|c|}
\hline & \multicolumn{3}{|l|}{ Small GO } & \multicolumn{3}{|l|}{ Large GO } \\
\hline & $\begin{array}{l}\text { Starting } \\
\text { material }\end{array}$ & $\begin{array}{l}\text { Hydrothermal } \\
\text { reduction }\end{array}$ & Epoxidation & $\begin{array}{l}\text { Starting } \\
\text { material }\end{array}$ & $\begin{array}{l}\text { Hydrothermal } \\
\text { reduction }\end{array}$ & Epoxidation \\
\hline $\begin{array}{l}\text { Non-functionalized } \\
\text { (C/O ratio) }\end{array}$ & $\mathrm{GO}_{\mathrm{S}}(2.2 \pm 0.1)$ & $\mathrm{rGO}_{\mathrm{S}}(3.5 \pm 0.2)$ & $\mathrm{rGO}_{\mathrm{s}}-\mathrm{O}_{3}(2.6 \pm 0.1)$ & $\mathrm{GO}_{\mathrm{L}}(2.6 \pm 0.1)$ & $\mathrm{rGO}_{\mathrm{L}}(6.1 \pm 0.1)$ & $\mathrm{rGO}_{\mathrm{L}}-\mathrm{O}_{3}(3.5 \pm 0.1)$ \\
\hline PEI-functionalized & $\mathrm{GO}_{\mathrm{S}}-\mathrm{PEI}$ & $\mathrm{rGO}_{\mathrm{S}}-\mathrm{PEI}$ & $\mathrm{rGO}_{\mathrm{S}}-\mathrm{O}_{3}-\mathrm{PEI}$ & $\mathrm{GO}_{\mathrm{L}}-\mathrm{PEI}$ & $\mathrm{rGO}_{\mathrm{L}}-\mathrm{PEI}$ & $\mathrm{rGO}_{\mathrm{L}}-\mathrm{O}_{3}-\mathrm{PEI}$ \\
\hline
\end{tabular}

zation, all prepared materials have been functionalized with polyethylenimine (PEI) via an epoxy ring opening reaction. ${ }^{25}$ In Table 1, the list of the different samples tested for siRNA binding is reported.

In order to measure the level of functionalization, we assessed the thermal profiles of the series of $\mathrm{GO}_{\mathrm{S}}$ and $\mathrm{GO}_{\mathrm{L}}$ by TGA (Fig. S2, ESI $\dagger$ ). ${ }^{24}$ The TGA curve of $\mathrm{GO}_{\mathrm{S}}$ [Fig. S2(a), ESI $\dagger$ ] shows the typical decomposition features of the GO material. The first mass decrease below $120{ }^{\circ} \mathrm{C}$ can be ascribed to the loss of physisorbed water molecules, while the significant mass loss between 120 and $250{ }^{\circ} \mathrm{C}$ is related to the decomposition of the oxygenated groups on the GO sheets. A similar trend can be seen in the TGA curves of $\mathrm{rGO}_{\mathrm{S}}$ and $\mathrm{rGO}_{\mathrm{S}}-\mathrm{O}_{3}$ [Fig. S2(b) and (c), respectively in the ESI $\dagger$, but with a different percentage in mass loss. Indeed, compared to $\mathrm{GO}_{\mathrm{S}}$, $\mathrm{rGO}_{\mathrm{S}}$ shows a less pronounced decomposition in the range between 120 and $250{ }^{\circ} \mathrm{C}$, while $\mathrm{rGO}_{\mathrm{S}}-\mathrm{O}_{3}$ mass loss, in the same temperature range, is between $\mathrm{rGO}_{\mathrm{S}}$ and $\mathrm{GO}_{\mathrm{S}}$. This behavior is related to the reduction/oxidation treatment performed on the sample. In fact, the hydrothermal process on $\mathrm{GO}_{\mathrm{S}}$ leads to oxygen depletion, while the $\mathrm{rGO}_{\mathrm{S}}$ epoxidation restores some oxygenated groups on the nanostructure as supported by the higher loss above $250{ }^{\circ} \mathrm{C}$ in the $\mathrm{rGO}_{\mathrm{S}}-\mathrm{O}_{3}$ thermogram. As previously described, a different trend was observed in the TGA curve in the case of $\mathrm{GO}_{\mathrm{L}}$ during the oxidation and reduction treatments. $^{24}$ Additional characterization was performed by XPS. This analysis (Table 1 and Fig. S3 and S4 in the ESI $\dagger$ ) showed similar values for both $\mathrm{GO}_{\mathrm{S}}$ and $\mathrm{GO}_{\mathrm{L}}$. The calculated $\mathrm{C} / \mathrm{O}$ ratio around 2.5 is typical of most of the GO materials. ${ }^{26}$ The hydrothermal reduction process allowed diminishing the oxygen content on the GO surface. In particular, the hydrothermal treatment of $\mathrm{rGO}_{\mathrm{S}}$ and $\mathrm{rGO}_{\mathrm{L}}$ afforded materials with $\mathrm{C} / \mathrm{O}$ ratios of 3.5 and 6.1 , respectively. The decrease of $\mathrm{O} \%$ is more evident in the case of $\mathrm{GO}_{\mathrm{L}}$ than in $\mathrm{GO}_{\mathrm{S}}$. This behavior may be attributed to a different reactivity due to the lateral size; more stable oxygenated groups such as carbonyl groups are predominant for $\mathrm{GO}_{\mathrm{S}}$. Ozone treatment caused a decrease of $\mathrm{C} / \mathrm{O}$ ratio to 2.6 in $\mathrm{rGO}_{\mathrm{S}}-\mathrm{O}_{3}$ and to 3.5 in $\mathrm{rGO}_{\mathrm{L}}-\mathrm{O}_{3}$, proving the successful epoxidation of the graphene sheets. Moreover, the increase in the $\mathrm{C}-\mathrm{O}$ band in the case of $\mathrm{rGO}_{\mathrm{S}}-\mathrm{O}_{3}$ (Fig. $\mathrm{S} 4 \mathrm{c}$ in the $\mathrm{ESI} \dagger$ ) and $\mathrm{rGO}_{\mathrm{L}}-\mathrm{O}_{3}{ }^{24}$ proved that the ozone treatment is able to introduce new epoxide groups on the GO surface.

Following the preparation and characterization of GO with different oxygen contents, we functionalized them with low molecular weight PEI to clarify the role of amino groups in terms of interaction with double strand siRNA. The thermal profile of the amino functionalized GO was assessed by TGA (Fig. S5, ESI $\dagger$ ). ${ }^{24}$ After PEI functionalization, all samples displayed a higher mass loss above $350{ }^{\circ} \mathrm{C}$ related to the degradation of the polymer chains. XPS analysis of PEI-functionalized GO was also performed. The appearance of a peak corresponding to N 1s (Fig. S3b in the ESI $\dagger$ ) suggested the successful amino derivatization of the GO sheets (Table S1 in the ESI $\dagger$ ). To better understand the effect of oxygenation and functionalization of GO, X-ray diffraction (XRD) characterization was conducted on the small $\mathrm{GO}_{\mathrm{S}}$ samples (Fig. S6 and Table S2 in the $\mathrm{ESI} \dagger)$. Starting $\mathrm{GO}_{\mathrm{S}}$ shows a peak centered at $2 \theta=11.6^{\circ}$ corresponding to a distance of $7.6 \AA$. This value is typical of GO nanostructures, ${ }^{27}$ and is attributed to the [002] interplanar distance in the GO lattice. Reduction and epoxidation processes led to a shift of the [002] XRD peak maximum up to $2 \theta=12.9^{\circ}$ and $12.8^{\circ}$ for $\mathrm{rGO}_{\mathrm{s}}$ and $\mathrm{rGO}_{\mathrm{s}}-\mathrm{O}_{3}$, respectively. The changes detected in the sheet to sheet distances can be ascribed to the variation in the oxygen content on the sample surface. ${ }^{27}$ Indeed, the hydrothermal reduction depletes oxygen from the GO surface causing a diminution in the average layer to layer distance to $6.8 \AA$, while $\mathrm{rGO}_{\mathrm{S}}$ epoxidation causes a small increase up to $6.9 \AA$. In the case of PEI functionalized $\mathrm{GO}_{\mathrm{S}}$ materials, the interplanar distance increased to $9.3 \AA$, which is attributed to the grafting of the polymer chains on the GO surface.

\subsection{Interaction of GO with SiRNA}

We previously proved that non-functionalized GO is able to adsorb siRNA molecules and that this interaction is driven by the oxygenated groups present on the GO surface. ${ }^{24}$ When GO is functionalized with PEI, the interaction is guided by the polymer chains and the residual oxygenated groups rather act to hamper the siRNA complexation. First, the complexation of siRNA with the non-functionalized $\mathrm{GO}_{\mathrm{S}}$ and $\mathrm{GO}_{\mathrm{L}}$ series and their derivatives, functionalized with PEI, was investigated using a gel retardation assay. For comparison with the series of $\mathrm{GO}_{\mathrm{L}}$, we used the same mass ratio of GO/siRNA at 0,50 , 100, 150, 200 and 300. The amount of free siRNA molecules was then estimated by comparing the fluorescence signal between the reference (containing only siRNA) and the other complexation ratios as shown in Fig. S7 (in the ESI $\dagger$ ) and in our previous work. ${ }^{24}$ In both the series of GO, the presence of PEI was able to induce a higher complexation capacity in comparison with the starting GO. Additional HPLC analyses (Fig. S8 in the ESI $\dagger$ ) proved that there is negligible denaturation of the duplex after the interaction of siRNA with $\mathrm{GO}_{\mathrm{S}}$. 
Adsorption of nucleic acids onto GO is a reversible process, therefore DNA or RNA molecules can easily adsorb onto and desorb from the GO surface (Fig. 1a). ${ }^{28}$

Similarly, siRNA can adsorb and desorb continuously from GO. To clarify the supramolecular interaction of GO with siRNA molecules, and in particular to understand if GO can alter the conformation of the native siRNA, we first studied the GO siRNA interaction via directly mixing the $\mathrm{GO}_{\mathrm{S}}$ with siRNA at a mass ratio of 2 . The siRNA conformational changes were followed using CD (Fig. 1b). This mass ratio was the maximum we could load in the CD cuvette without reaching absorbance saturation and still appreciating the siRNA eventual changes. Surprisingly, an intense bathochromic shift and a clear decrease of the siRNA signal at $264 \mathrm{~nm}$ were observed after 2 hours of incubation. This change cannot be ascribed to a substantial adsorption of siRNA onto the GO surface. Indeed, as proved by electrophoresis, good complexation occurs at a much higher mass ratio (Fig. S7 in the ESI†). We can attribute this relevant change in the $\mathrm{CD}$ spectral characteristics of SiRNA to the dynamic interaction of GO with the duplex. Based on these initial results, we decided to understand what would happen to siRNA molecules under real complexation conditions at much higher GO concentrations. For this purpose, we had to overcome some spectroscopic characteristics of GO: for instance, GO displays a high absorbance below $300 \mathrm{~nm}$ in the UV-Vis spectra. ${ }^{29}$ In addition, GO is a
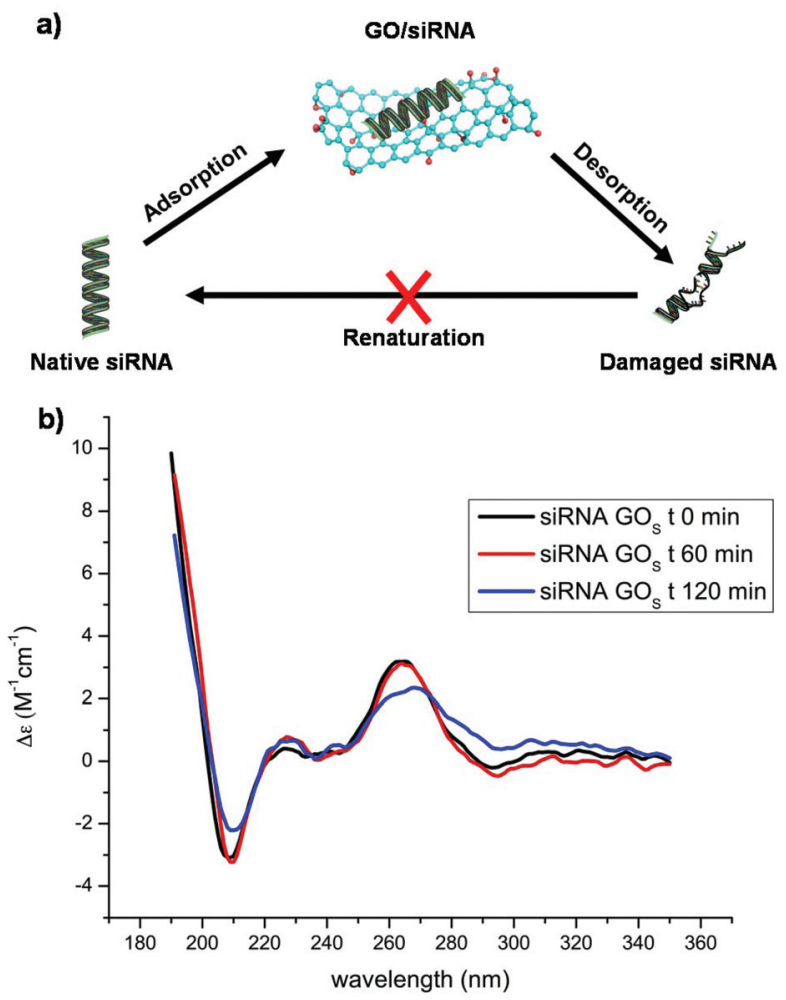

Fig. 1 (a) Scheme of the dynamic equilibrium between the adsorption and desorption of siRNA onto the GO surface. (b) CD spectra of the siRNA mixed with $\mathrm{GO}_{S}$ recorded at different time points. good acceptor that can efficiently quench dyes and therefore may interfere with many optical spectroscopies. Such interference makes the study of the interaction between GO and other molecules complicate. For these reasons, we decided to investigate the siRNA conformational changes after removing the GO from the solution.

More precisely, after performing the incubation of siRNA with GO, we filtered the GO complex and collected the free siRNA molecules. With this method, we were able to collect and analyze the siRNA molecules in dynamic equilibrium with the GO materials after the incubation, and therefore to indirectly evaluate the siRNA/GO interaction (Fig. 1a). Initially, we optimized the conditions in order to avoid the passage of the different GO and GO-PEI conjugates through the filters (data not shown), measuring the absorbance at $230 \mathrm{~nm}$ of each GO dispersion after filtration. In addition, we demonstrated that siRNA molecules are able to easily pass through the filters by measuring their absorbance at $260 \mathrm{~nm}$ before and after filtration. Filtered siRNA solutions have also been used as a reference for all the experiments to avoid any effect of this treatment on the siRNA conformational stability. The GO/siRNA mass ratio was another crucial parameter to take into consideration. Indeed, a low mass ratio may not induce significant changes in the siRNA structure, while a too high mass ratio would favor the complexation leaving a very low amount of free siRNA passing through the filter, likely not detectable by UV-Vis. Generally, the GO/siRNA mass ratio used for in vitro experiments is between 10 and $50 .{ }^{30}$ We decided to perform our experiments at a mass ratio of 20 . This value reflects a good compromise between the complexing ratios found in the literature and the scope of our study. Only $\mathrm{rGO}_{\mathrm{L}}-$ PEI was used at a mass ratio of 5 because of its high siRNA complexation ability.

Considering all these parameters, we initially analyzed the UV-Vis spectra of free siRNA after complexing with different GO materials. The absorbance bands of dsRNA are very sensitive to intramolecular $\mathrm{H}$-bonding cleavage. ${ }^{31}$ In particular, it was reported that the nucleotide unpairing in dsRNA nucleobases induces a hyperchromic effect on the band centered at $260 \mathrm{~nm}$ that can be easily detected via UV-Vis spectroscopy. To study if our GO materials were able to induce unpairing of nucleobases, we used siRNA labeled with the Yakima Yellow (YY) dye.

The calibration curve of siRNA was obtained using the YY absorbance at $522 \mathrm{~nm}$ and the nucleobase absorbance at $260 \mathrm{~nm}$ (Fig. S9 in the ESI $\dagger$ ). The YY absorbance is proportional to the real concentration of siRNA molecules in solution while the siRNA absorbance at $260 \mathrm{~nm}$ may be affected by the base unpairing. ${ }^{31}$ After interaction with the different GO materials, the free siRNA was collected and analyzed by UV-Vis. The concentration of free siRNA was measured using the YY absorbance. The hyperchromicity was calculated considering the percentage of increase of the siRNA absorbance at $260 \mathrm{~nm}$ after making contact with GO and the siRNA absorbance, at the same concentration, estimated from the YY calibration curve. Fig. 2 clearly shows the hyperchromic effect 


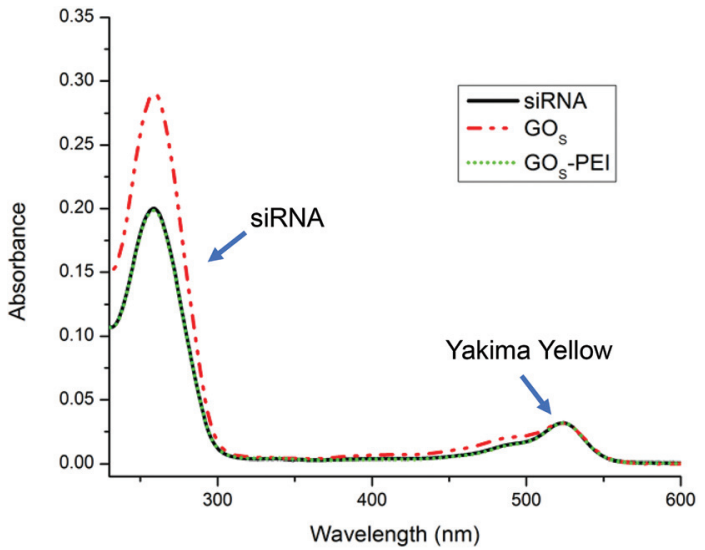

Fig. 2 UV-Vis spectra at the same concentration (corresponding to the same absorbance intensity at $522 \mathrm{~nm}$ ) of the starting siRNA (black line), siRNA after incubation with $\mathrm{GO}_{\mathrm{S}}$ (dashed-dotted red line) and siRNA after incubation with $\mathrm{rGO}_{\mathrm{S}}-\mathrm{PEI}$ (dotted green line).

occurring when siRNA is in contact with $\mathrm{GO}_{\mathrm{S}}$, while the functionalization with PEI does not affect the intensity of the maximum at $260 \mathrm{~nm}$. A similar trend was also observed for the $\mathrm{GO}_{\mathrm{L}}$ series (data not shown).

The data, obtained from the UV-Vis analysis, for the different GO materials are summarized in Fig. 3. Non-functionalized small GO series $\left(\mathrm{GO}_{\mathrm{S}}, \mathrm{rGO}_{\mathrm{S}}\right.$ and $\left.\mathrm{rGO}_{\mathrm{s}}-\mathrm{O}_{3}\right)$ induced a high hyperchromicity reaching intensities higher than 55\%, without an appreciable difference among them (Fig. 3a).

Similar hyperchromicity was observed by measuring the solution after 6 hours from the filtration indicating that the damage on siRNA induced by the $\mathrm{GO}_{\mathrm{S}}$ series is permanent (not shown). PEI functionalization of small GO samples was able instead to remarkably reduce the hyperchromic effect. Similar results were found for the large GO samples. Non-functionalized $\mathrm{GO}_{\mathrm{L}}, \mathrm{rGO}_{\mathrm{L}}$ and $\mathrm{rGO}_{\mathrm{L}}-\mathrm{O}_{3}$ showed a hyperchromic effect higher than $25 \%$ (Fig. 3b). Even in this case, the hyperchromic effect was almost completely suppressed after PEI functionalization. On comparison of the non-functionalized $\mathrm{GO}_{\mathrm{S}}$ and $\mathrm{GO}_{\mathrm{L}}$ samples, we can see that small GO destabilizes the double strands of siRNA more than large GO. This behavior can be attributed mainly to the effect of size. Indeed, it has already been demonstrated that small GO can intercalate into DNA breaking the $\mathrm{H}$-bonds inside the double strands. ${ }^{21,22}$ Most probably, smaller GO flakes can intercalate more easily into DNA nucleobases than larger GO, thus inducing more damage on the double strand structure. There are no evident trends comparing the amount of oxygenated groups on the GO surface. Intercalation seems instead driven by the GO size.

Interestingly, PEI functionalized GO samples exhibit a negligible $\mathrm{H}$-bonding cleavage with a hyperchromic percentage lower than $5 \%$. This effect is likely due to the direct interaction of PEI chains that protect the integrity of the siRNA double strand. ${ }^{24}$

To better elucidate the mechanism of the interaction between the small and large GO materials, we next tested the
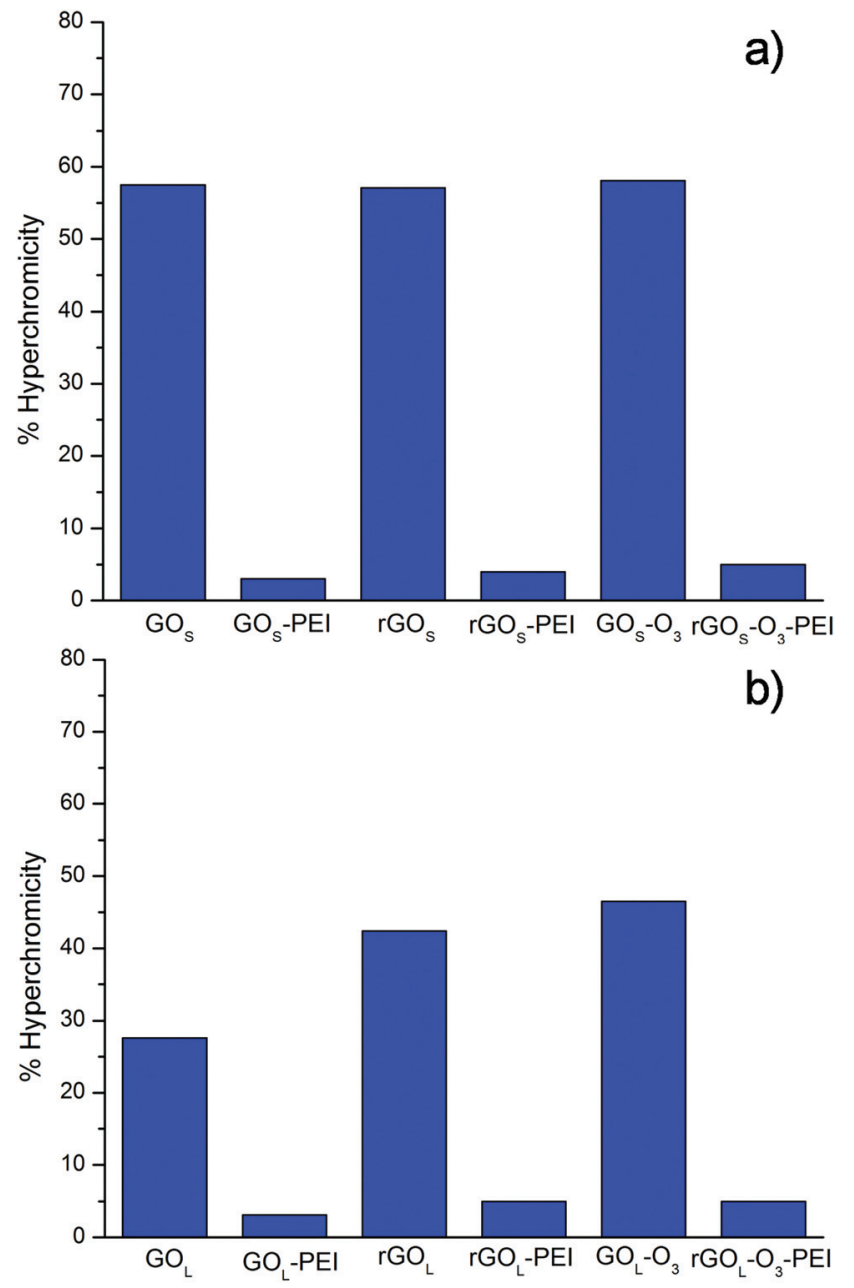

Fig. 3 Hyperchromicity values of siRNA at $260 \mathrm{~nm}$ after incubation with the different $\mathrm{GO}$ conjugates. The reported data are obtained from two sets of measurements.

integrity of free siRNA using FRET probes. For this characterization, we used a double strand siRNA (termed $5^{\prime}-5^{\prime}$ ) with one strand labeled at the $5^{\prime}$ position with Alexa Fluor ${ }^{\circledR 546}$ (donor) and the other strand labeled at the $5^{\prime}$ position with Alexa Fluor ${ }^{\circledR 647}$ (acceptor), respectively. As a donor alone, we used a siRNA functionalized at the $5^{\prime}$ position with Alexa Fluor ${ }^{\circledR} 546$. With this donor/acceptor pair we were able to estimate the $5^{\prime}-5^{\prime}$ distance (longitudinal distance) of the siRNA chain (Table 2). Another important information was to understand if the strand to strand distance may be affected by the GO interaction. For these sets of experiments, we decided to use a second double strand siRNA (termed $3^{\prime}-5^{\prime}$ ) labeled at the $5^{\prime}$ position with $C y \circledast 3$ (donor) and the other strand marked at the $3^{\prime}$ position with $\mathrm{Cy} \circledast 5$ (acceptor), respectively. As a donor alone, we used a dsRNA functionalized at the $5^{\prime}$ position with Cy®3 (Table 2). Each FRET pair and the solution of donor alone have been incubated with $\mathrm{GO}$ at a GO/siRNA mass ratio of 20 . After $30 \mathrm{~min}$, the solutions were filtered and analyzed via UV-Vis and fluorescence spectroscopy techniques (Fig. S10 
Table 2 Distances measured via FRET on siRNA after interaction with the different GO: $3^{\prime}-5^{\prime}$ and $5^{\prime}-5^{\prime}$ distance corresponds to the distance between Alexa Fluor ${ }^{2} 46 /$ Alexa Fluor $\AA 647$ and $C y \circledast 3 / C y \circledast 5$ labeled siRNA pairs, respectively

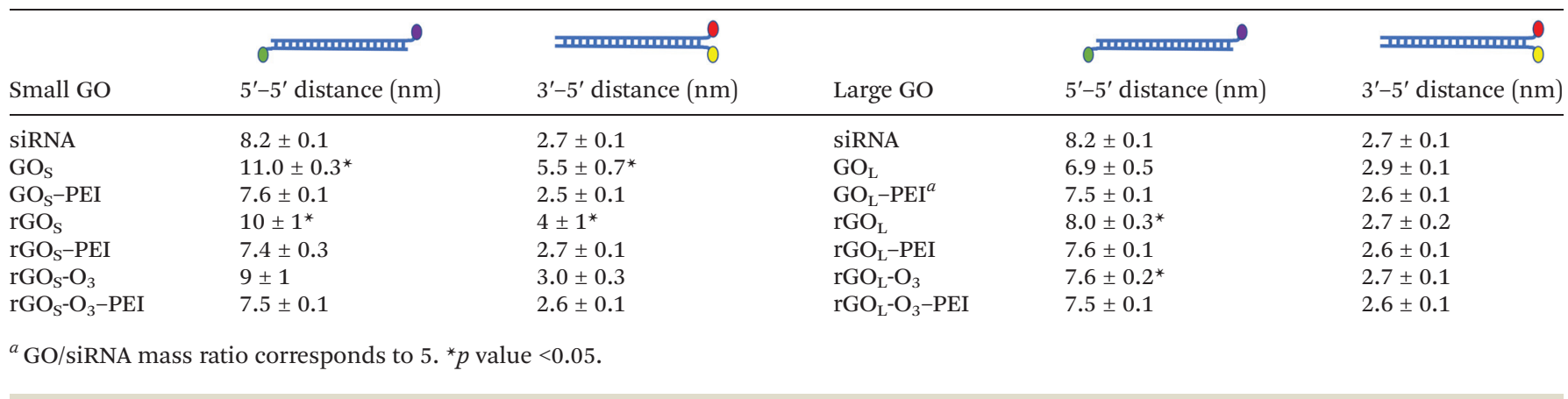

in the ESI $\dagger$ ). FRET efficiency $(E)$ was calculated using eqn (1) and (2) given in the ESI. $\dagger^{32}$ The Förster radius was assumed to be $3.8 \mathrm{~nm}$ for the couple $\mathrm{Cy} \otimes 3 / \mathrm{Cy}{ }^{\circledR} 5^{32}$ and $7.4 \mathrm{~nm}$ for the Alexa Fluor ${ }^{\circledR 546 / A l e x a}$ Fluor $₫ 647$ as reported from the provider. ${ }^{33}$ Since the FRET efficiency is inversely proportional to the sixth power of the distance $R$ between the donor and the acceptor, the two fluorescent molecules should be in close proximity to allow significant energy transfer. The measured distances are given in Table 2.

A siRNA molecule can be represented as a nanometric rod with an average size below $10 \mathrm{~nm}$ in length and approximately $2 \mathrm{~nm}$ in diameter. ${ }^{34}$ FRET measurement of native siRNA used in our experiments showed a longitudinal distance of $8.2 \pm$ $0.1 \mathrm{~nm}$ (measuring the $5^{\prime}-5^{\prime}$ distance) and a strand to strand distance of $2.7 \pm 0.1 \mathrm{~nm}$ (measuring the $3^{\prime}-5^{\prime}$ distance) (Table 2). These values are in full agreement with those found in the literature. ${ }^{34}$ The non-functionalized small GO samples display a general enhancement of both lateral and longitudinal distances. In particular, the $5^{\prime}-5^{\prime}$ distance was $11.0 \pm 0.3,10 \pm 1$ and $9 \pm 1 \mathrm{~nm}$ for $\mathrm{GO}_{\mathrm{S}}, \mathrm{rGO}_{\mathrm{S}}$ and $\mathrm{rGO}_{\mathrm{S}}-\mathrm{O}_{3}$, respectively. The $3^{\prime}-5^{\prime}$ distance also increased to $5.5 \pm 0.7 \mathrm{~nm}$ for $\mathrm{GO}_{\mathrm{S}}$, and to $4 \pm 1$ and $3.0 \pm 0.3 \mathrm{~nm}$ for $\mathrm{rGO}_{\mathrm{S}}$ and $\mathrm{rGO}_{\mathrm{S}}-\mathrm{O}_{3}$, respectively. No significant differences were instead observed for PEI functionalized small $\mathrm{GO}_{\mathrm{S}}$ in the $\mathrm{CC}$ distance, meanwhile the $5^{\prime}-5^{\prime}$ distance appears a bit shorter (i.e. 0.6-0.8 nm) (Table 2). In the case of large $\mathrm{GO}_{\mathrm{L}}$, less evident changes in the strand-to-strand and the longitudinal distances could be observed. The data for $\mathrm{GO}_{\mathrm{L}}, \mathrm{rGO}_{\mathrm{L}}$ and $\mathrm{rGO}_{\mathrm{L}}-\mathrm{O}_{3}$ correspond to $6.9 \pm 0.5,8.0 \pm 0.3$ and $7.6 \pm 0.2 \mathrm{~nm}$ for the $5^{\prime}-5^{\prime}$ distances, and to $2.9 \pm 0.1,2.7 \pm 0.2$ and $2.7 \pm 0.1 \mathrm{~nm}$ for the $3^{\prime}-5^{\prime}$ distances, respectively. Even in the case of large $\mathrm{GO}_{\mathrm{L}}$ flakes functionalized with PEI, the interaction with siRNA did not affect the lateral distances, but slightly decreases the longitudinal distance $(7.5 \pm 0.1 \mathrm{~nm}$ for $\mathrm{GO}_{\mathrm{L}}-\mathrm{PEI}$ and $\mathrm{rGO}_{\mathrm{L}}-\mathrm{O}_{3}-\mathrm{PEI}$, and $7.6 \pm 0.1 \mathrm{~nm}$ for $\mathrm{rGO}_{\mathrm{L}}-\mathrm{PEI}$ ). FRET distances have been calculated from an average of at least two different experiments in each measurement. To validate our data, we performed statistical treatment by comparing the FRET distances found on native siRNA with the distances obtained from siRNA incubated with the most representative non-functionalized GO materials. The results confirm that the measured FRET differences are statistically significant.
As mentioned before, hyperchromicity analysis gave us some information about the H-bond breaking, while FRET helped to understand the displacement of the RNA strands after the interaction with GO. However, neither FRET nor UV allows us to describe the secondary structure of the siRNA. To deeply understand the nature of the structural modifications induced by GO, we decided to characterize the siRNA conformation after incubating with GO using circular dichroism. In Fig. 4 , we show the CD spectra of the siRNA after the process of incubation with GO and filtration.

The native siRNA CD spectrum (Fig. 4, black lines) shows a positive band at $264 \mathrm{~nm}$ and a negative band at $210 \mathrm{~nm}$, which are typical of an A-helix conformation. ${ }^{35,36}$ In particular, the positive band at $264 \mathrm{~nm}$ is associated with the stacking interaction between the planar base pairs and the helical structure of the RNA that induces a chiral environment on the nucleobases, while the negative band centered at $210 \mathrm{~nm}$ is related to the A-helix backbone conformation..$^{37}$ After contact with small $\mathrm{GO}_{\mathrm{S}}$ flakes, several changes in the $\mathrm{CD}$ spectra can be observed (Fig. 4a). The positive band at $264 \mathrm{~nm}$ decreases in intensity with a bathochromic shift of the maximum, while the negative band centered at $210 \mathrm{~nm}$ is remarkably reduced in intensity. Comparing the shifts of the positive band, the siRNA signal undergoes a shift of $\approx 15 \mathrm{~nm}$ after the interaction with $\mathrm{GO}_{\mathrm{S}}$, while with $\mathrm{rGO}_{\mathrm{S}}$ and $\mathrm{rGO}_{\mathrm{s}}-\mathrm{O}_{3}$ the shifts are about $7 \mathrm{~nm}$. This behavior is typically related to the siRNA unfolding and denaturation. $^{35,38}$ Instead, the interaction of siRNA with PEI functionalized $\mathrm{GO}_{\mathrm{S}}$ does not alter the polynucleotide secondary structure (Fig. 4b) showing only a small decrease of the band at $210 \mathrm{~nm}$. In the case of non-functionalized $\mathrm{GO}_{\mathrm{L}}$ samples, the CD spectra appear different (Fig. 4c). Once again, the siRNA secondary structure is affected. We measured an enhancement of the band at $264 \mathrm{~nm}$ without any significant shift and a sensible decrease of the negative band at $210 \mathrm{~nm}$. It has been reported before that in duplex RNA, the CD intensity near $260 \mathrm{~nm}$ is inversely correlated with the helical winding angle ${ }^{39}$ Similarly, the increase in the CD intensity induced by the interaction of the $\mathrm{GO}_{\mathrm{L}}$ series can be associated with a reduction of the winding angle of the siRNA helix, leading to a modification of the structure in close relation with the FRET analysis. siRNA treated with the PEI functionalized large $\mathrm{GO}_{\mathrm{L}}$ 

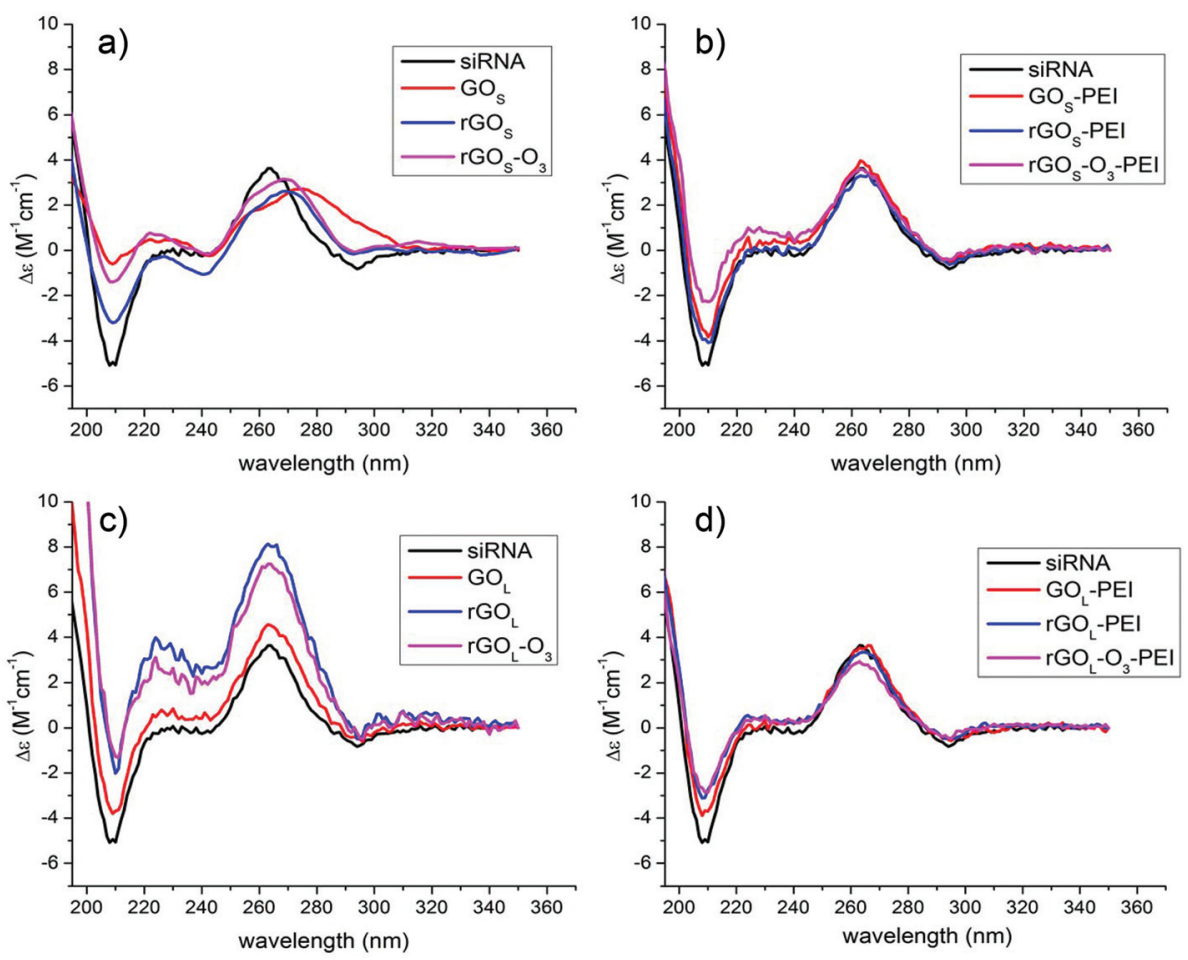

Fig. $4 \mathrm{CD}$ spectra of siRNA after incubation and filtration with a series of: (a) $\mathrm{GO}_{\mathrm{S}}$ (b) $\mathrm{GO}_{\mathrm{S}}-\mathrm{PEI}$, (c) $\mathrm{GO}_{\mathrm{L}}$, and (d) $\mathrm{GO}_{\mathrm{L}}-\mathrm{PEI}$, respectively.

series presents only small modifications in the $210 \mathrm{~nm} \mathrm{CD}$ band like in the case of the PEI functionalized small $\mathrm{GO}_{\mathrm{S}}$ materials (Fig. 4d). All data on the siRNA conformation from CD spectroscopy confirmed strong conformational changes due to the interaction with the different non-functionalized GO materials.

Combining the results from hyperchromism, FRET and CD, we can conclude that siRNA presents clear structural modifications after interaction with GO, induced by both small and large sized nanosheets. The nature and the type of interaction are however dependent on the GO size. Comparing the two series of non-functionalized small and large GO, the $\mathrm{GO}_{\mathrm{S}}$ series shows a higher hyperchromicity with an enhancement of the lateral and the longitudinal distances. The changes in these parameters demonstrate unambiguously that smaller GO is able to induce more damage in the siRNA structure than the larger one. It has been already reported that GO causes the cleavage of the DNA duplex. ${ }^{21}$ In our study, HPLC analysis (Fig. S8 in the ESI†) showed that the two siRNA strands are still intramolecularly stabilized. In addition, the CD analysis showed that incubation with $\mathrm{GO}_{\mathrm{S}}$ samples sensibly decreases the helicity. These results clearly suggest that double strand denaturation occurs, without necessarily leading to complete strand displacement or duplex breakage. ${ }^{35,38}$ On the other hand, the mechanism of interaction is not easy to assess. It has been already supposed that GO can intercalate between the nucleobases. ${ }^{21}$ However, this mechanism should be better elucidated since there is a remarkable size difference between the GO and the siRNA duplex. Besides, the small $\mathrm{GO}_{\mathrm{S}}$ flakes leading to a strong unpairing (confirmed by high hyperchromicity) cause a moderate displacement of the two strands (confirmed by higher FRET distances) and induce a partial transition from the A-helix to the random coil (lower and shifted helicity at $264 \mathrm{~nm}$ ). This siRNA damage provoked by the $\mathrm{GO}_{\mathrm{S}}$ series does not seem to be induced by simple adsorption but mostly by a partial insertion (from the edges) of the small flakes within the double strand (Fig. 5a). We think that most probably small flake edges are able to interact with the siRNA nucleobases provoking partial interstrand $\mathrm{H}$-bond breaking.

Within the three small GO specimens, $\mathrm{GO}_{\mathrm{S}}$ shows the highest siRNA destabilization ability, while $\mathrm{rGO}_{\mathrm{S}}-\mathrm{O}_{3}$ induces more alterations in the native structure than $\mathrm{rGO}_{\mathrm{S}}\left(\mathrm{GO}_{\mathrm{S}}>\right.$ $\mathrm{rGO}_{\mathrm{S}}-\mathrm{O}_{3}>\mathrm{rGO}_{\mathrm{S}}$ ). This trend can be explained if we consider the functional groups present on the GO surface. In particular, this seems to be correlated with the $\mathrm{C} / \mathrm{O}$ ratio obtained from XPS (Table 1). Destabilization of siRNA with small GO may be trigged by the oxygenated groups. Most probably, these electronegative groups are able to form polar bonds with the nitrogen nucleobases, destabilizing the H-bond between the double strands. In the case of the large GO series, there is a lower destabilization of the interstrand $\mathrm{H}$-bonds (supported by low hyperchromicity) and a general reduction of the $5^{\prime}-5^{\prime}$ distance proved by FRET. On the other hand, CD spectra show an enhancement of the helicity at $264 \mathrm{~nm}$ due to a clear interaction with the large $\mathrm{GO}_{\mathrm{L}}$ flakes. Most probably, large $\mathrm{GO}_{\mathrm{L}}$ is too big to efficiently intercalate between the nucleotides, but it 
a)

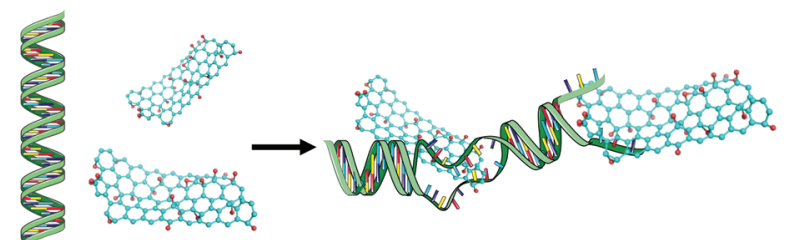

SIRNA

$\mathrm{GO}_{\mathrm{S}}$
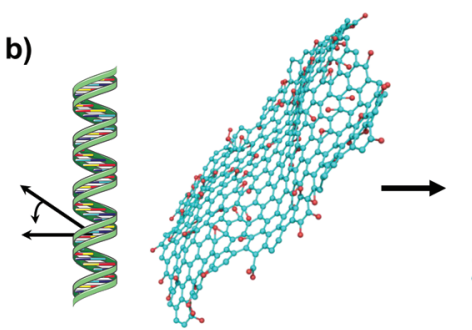

Helix denaturation

SIRNA

Winding angle perturbation
Fig. 5 Cartoon illustrating the possible interactions and conformational changes of (a) $\mathrm{GO}_{\mathrm{S}}$ and (b) $\mathrm{GO}_{\mathrm{L}}$ with the siRNA molecule.

interacts with the RNA groove causing a decrease of the winding angle, thus altering the structure of the double A-helix (Fig. 5b).

The reduction of the winding angle allows the increase of the number of bases per turn in the helix. This conformational change causes the enhancement of the base to base stacking (increasing the $\mathrm{CD}$ band at $264 \mathrm{~nm}$ ), alters the helix backbone structure (decreasing the $\mathrm{CD}$ band at $210 \mathrm{~nm}$ ), and provokes a helical length shrinking (as shown by FRET analysis). In the case of protein interaction with RNA, it has been proven, by solving the crystal structure of the complex, that the distortion from the ideal RNA A-helix is due to an interaction on the major groove without any contact with the nucleotides. ${ }^{40}$ For the large GO, there is destabilization of some H-bonds between the strands (Fig. 2), but it is not clear if this behavior is due to a direct interaction of GO with the nucleobases or only with the helix groove. Comparing the differently oxygenated large GO specimens, it appears clear that the $\mathrm{rGO}_{\mathrm{L}}-\mathrm{O}_{3}$ and $\mathrm{rGO}_{\mathrm{L}}$ induce more changes in the native siRNA conformation than $\mathrm{GO}_{\mathrm{L}}$. The latter tendency may be again correlated with the amount of oxygenated groups (Table 1). In particular, it seems that the oxygenated species avoid the siRNA conformational change. Most probably, the siRNA secondary structure alteration induced by large GO flakes is mediated by nonpolar interactions such as $\pi-\pi$ stacking. Interestingly, PEI functionalization of both large and small GO does not induce significant changes in the siRNA structure. Only small backbone conformational alterations (slight decrease of $210 \mathrm{~nm} \mathrm{CD}$ band) were evidenced, most probably due to the interaction between the positively charged PEI chains and the negative phosphate groups in the siRNA skeletal structure. ${ }^{41}$ However, these small changes are not yet clear. We hypothesized that the ionic interaction of PEI chains with the phosphate groups partially alters the siRNA backbone geometry causing a small
A-helix bending that provokes a decrease of the band at $210 \mathrm{~nm}$ in the CD spectra and the small shortening of the $5^{\prime}-5^{\prime}$ distance as seen by FRET. Nevertheless, these siRNA small secondary structure modifications have already been described in the literature and should not alter the siRNA biological activity in vitro or in vivo. ${ }^{41}$

\section{Conclusions}

In this work, we analyzed the siRNA structure alterations induced by GO. We performed a systematic study by varying the size and the oxidation level of GO from two different sources. In addition, to observe the effect of amino groups we tested different GO materials after PEI functionalization, and complexed with siRNA molecules. Using a mild filtration protocol, we were able to separate free siRNA molecules from GO flakes after their incubation and to assess the conformational damage via UV-Vis, fluorescence and CD spectra. A deep understanding of the interaction of siRNA and GO is crucial for its future applications. Our results indicate that the alteration of the siRNA structure is strongly mediated by the GO size. Indeed, small GO seems to intercalate between the double strands and induce severe damage on the RNA conformation. Large GO instead seems to basically reduce the A-helix pitch. In addition, we proved that even after 6 hours the siRNA was not able to recover its native structure. Interestingly, all the PEI functionalized GO materials did not show significant conformational alterations. We believe that the full comprehension of the supramolecular interactions between GO and siRNA is essential for an effective GO application in nanomedicine. Depending on the size, GO can efficiently unwind, displace and partially denature the double siRNA strands, thus likely altering its biological activity. For these reasons, we wish to suggest not to use non-functionalized GO in gene delivery or silencing. On the other hand, the results also demonstrate that PEI functionalized GO can overcome the partial denaturation effect of GO after interaction with siRNA, and retain the native oligonucleotide conformation. This protecting activity together with its high siRNA complexing capacity makes GOPEI a promising material for gene delivery and silencing applications.

\section{Conflicts of interest}

All the authors declare no conflicts of interest with this work.

\section{Acknowledgements}

The authors gratefully acknowledge the financial support from the EU H2020-Adhoc-2014-20 GrapheneCore1 (no. 696656) and from the Agence Nationale de la Recherche (ANR) through the LabEx project Chemistry of Complex Systems (ANR-10LABX-0026_CSC). This work was partly supported by the Centre National de la Recherche Scientifique (CNRS), the 
International Center for Frontier Research in Chemistry (icFRC), the JST PRESTO, and the JSPS KAKENHI (Science of Atomic Layers (SATL), Grant number 16H00915). N. D. Q. C. is grateful to Honda foundation for mobility grant to Japan. The authors thank Dr Serana Bernacchi for help in fluorescence spectroscopy measurements, Fanny Bonachera for preparing the GO models and Adriano Aloisi for HPLC analyses.

\section{References}

1 I. M. Verma and N. Somia, Nature, 1997, 389, 239-242.

2 H. Yin, R. L. Kanasty, A. A. Eltoukhy, A. J. Vegas, J. R. Dorkin and D. G. Anderson, Nat. Rev. Genet., 2014, 15, 541-555.

3 G. J. Hannon, Nature, 2002, 418, 244-251.

4 A. Fire, S. Xu, M. K. Montgomery, S. A. Kostas, S. E. Driver and C. C. Mello, Nature, 1998, 391, 806-811.

5 D. M. Dykxhoorn, D. Palliser and J. Lieberman, Gene Ther., 2006, 13, 541-552.

6 S. M. Elbashir, J. Harborth, W. Lendeckel, A. Yalcin, K. Weber and T. Tuschl, Nature, 2001, 411, 494-498.

7 K. A. Whitehead, R. Langer and D. G. Anderson, Nat. Rev. Drug Discovery, 2009, 8, 129-138.

8 M. Nurunnabi, K. Parvez, V. Revuri, H. A. Khan, X. Feng and Y. Lee, RSC Adv. , 2015, 5, 42141-42161.

9 Y. Zhang, T. R. Nayak and W. Cai, Nanoscale, 2012, 4, 38333842.

10 K. Yang, L. Feng, X. Shi and Z. Liu, Chem. Soc. Rev., 2013, 42, 530-547.

11 R. Kurapati, J. Russier, M. A. Squillaci, E. Treossi, C. MénardMoyon, A. E. Del Rio-Castillo, E. Vazquez, P. Samorì, V. Palermo and A. Bianco, Small, 2015, 11, 3985-3994.

12 D. R. Dreyer, S. Park, C. W. Bielawski, R. S. Ruoff, J. M. Tour, K. Müllen, A. Ivaska, R. G.-S. Goh, R. H. Friend, A. T. S. Wee, P. K.-H. Ho, D. Chen and R. S. Ruoff, Chem. Soc. Rev., 2010, 39, 228-240.

13 Y. Yang, A. M. Asiri, Z. Tang, D. Du and Y. Lin, Biochem. Pharmacol., 2013, 16, 365-373.

14 K. V. Krishna, C. Ménard-Moyon, S. Verma and A. Bianco, Nanomedicine, 2013, 8, 1669-1688.

15 J. Lee, Y. Yim, S. Kim, M. H. Choi, B. S. Choi, Y. Lee and D. H. Min, Carbon, 2016, 97, 92-98.

16 L. Tang, Y. Wang and J. Li, Chem. Soc. Rev., 2015, 44, 69546980.

17 H. Lei, L. Mi, X. Zhou, J. Chen, J. Hu, S. Guo and Y. Zhang, Nanoscale, 2011, 3, 3888-3892.

18 M. Liu, H. Zhao, S. Chen, H. Yu and X. Quan, Chem. Commun., 2012, 48, 564-566.
19 L. Tang, H. Chang, Y. Liu and J. Li, Adv. Funct. Mater., 2012, 22, 3083-3088.

20 X. Zhao, J. Phys. Chem. C, 2011, 115, 6181-6189.

21 H. Ren, C. Wang, J. Zhang, X. Zhou, D. Xu, J. Zheng, S. Guo and J. Zhang, ACS Nano, 2010, 4, 7169-7174.

22 B. Zheng, C. Wang, C. Wu, X. Zhou, M. Lin, X. Wu, X. Xin, X. Chen, L. Xu, H. Liu, J. Zheng, J. Zhang and S. Guo, J. Phys. Chem. C, 2012, 116, 15839-15846.

23 N. Morimoto, T. Kubo and Y. Nishina, Sci. Rep., 2016, 6, 21715.

24 N. D. Q. Chau, G. Reina, J. Raya, I. A. Vacchi, C. MénardMoyon, Y. Nishina and A. Bianco, Carbon, 2017, 122, 643652.

25 I. A. Vacchi, C. Spinato, J. Raya, A. Bianco and C. MénardMoyon, Nanoscale, 2016, 8, 13714-13721.

26 S. Makharza, G. Cirillo, A. Bachmatiuk, I. Ibrahim, N. Ioannides, B. Trzebicka, S. Hampel and M. H. Rümmeli, J. Nanopart. Res., 2013, 15, 2099.

27 N. Morimoto, T. Kubo and Y. Nishina, Sci. Rep., 2016, 6, 21715.

28 C. H. Lu, H. H. Yang, C. L. Zhu, X. Chen and G. N. Chen, Angew. Chem., Int. Ed., 2009, 48, 4785-4787.

29 J. Shang, L. Ma, J. Li, W. Ai, T. Yu and G. G. Gurzadyan, Sci. Rep., 2012, 2, 792.

30 G. Shim, M.-G. Kim and J. Y. Park, Adv. Drug Delivery Rev., 2016, 105, 205-227.

31 D. E. Draper, Y. Xing and L. G. Laing, J. Mol. Biol., 1995, 249, 231-238.

32 M. Massey, W. R. Algar and U. J. Krull, Anal. Chim. Acta, 2006, 568, 181-189.

33 https://www.thermofisher.com/uk/en/home/references/molecular-probes-the-handbook/tables/r0-values-for-some-alexafluor-dyes.html.

34 J. M. Barichello, S. Kizuki, T. Tagami, L. A. L. Soares, T. Ishida, H. Kikuchi and H. Kiwada, Int. J. Pharm., 2012, 430, 359-365.

35 J. Kypr, I. Kejnovská, D. Renciuk and M. Vorlícková, Nucleic Acids Res., 2009, 37, 1713-1725.

36 I. S. Cho, J. Kim, D. H. Lim, H.-C. Ahn, H. Kim, K.-B. Lee and Y. S. Lee, Biotechnol. Lett., 2008, 30, 1901-1908.

37 R. W. Woody, Methods Enzymol., 1995, 246, 34-71.

38 D. J. Phillips and A. M. Bobst, Biochem. Biophys. Res. Commun., 1972, 47, 150-156.

39 J. W. Ucci, Y. Kobayashi, G. Choi, A. T. Alexandrescu and J. L. Cole, Biochemistry, 2007, 46, 55-65.

40 J. M. Ryter and S. C. Schultz, EMBO J., 1998, 17, 7505-7513.

41 O. M. Merkel, A. Beyerle, D. Librizzi, A. Pfestroff, T. M. Behr, B. Sproat, P. J. Barth and T. Kissel, Mol. Pharm., 2009, 6, 1246-1260. 